\title{
CAPE promotes TRAIL-induced apoptosis through the upregulation of TRAIL receptors via activation of $p 38$ and suppression of JNK in SK-Hep1 hepatocellular carcinoma cells
}

\author{
EUN YOUNG KIM, JAE-HA RYU and AN KEUN KIM \\ College of Pharmacy, Sookmyung Women's University, Seoul 140-742, Republic of Korea
}

Received May 2, 2013; Accepted June 21, 2013

DOI: $10.3892 /$ ijo.2013.2018

\begin{abstract}
Caffeic acid phenethyl ester (CAPE), a phenolic compound derived from honeybee propolis, has been reported to possess anticancer activities in several types of malignant cells. Here, we show that treatment with tumor necrosis factor-related apoptosis-inducing ligand (TRAIL) in combination with CAPE significantly sensitized SK-Hep1 cells to TRAIL-induced apoptosis. The sensitization to TRAIL was accompanied by the activation of extrinsic and intrinsic apoptotic pathways, leading to the activation of caspases, mitochondrial disruption and PARP cleavage. Moreover, TRAIL receptors, such as DR4 and DR5 were significantly upregulated by CAPE treatment, and both DR4/Fc and DR5/Fc chimera markedly abrogated apoptosis induced by CAPE and TRAIL, demonstrating the critical role of these death receptors in combination-induced apoptosis. The effect of CAPE on mitogen-activated protein kinases (MAPKs) was further examined, where CAPE treatment resulted in the activation of $\mathrm{p} 38$ and the inhibition of JNK, without affecting levels of phospho-ERK. Our results showed that p38 and JNK exhibited the opposite role in SK-Hepl cells. The inhibition of p38, using SB203580, blocked the CAPE-induced expression of death receptors and attenuated the combination-induced apoptosis, suggesting the pro-apoptotic role of p38. In contrast, JNK-specific inhibition, by SP600125, triggered upregulation of DR4 and DR5, and sensitized SK-Hep1 cells to TRAIL, indicating that the CAPE-induced suppression of JNK may contribute to the sensitizing effect of CAPE through the upregulation of death receptors. Taken together, these results indicate that CAPE potentiated TRAIL-induced apoptosis in SK-Hep1 cells, through upregulation of TRAIL receptors via modulation of $\mathrm{p} 38$ and JNK signaling pathways.
\end{abstract}

Correspondence to: Professor An Keun Kim, College of Pharmacy, Sookmyung Women's University, Hyochanwon-Gil 52, Yongsan-Ku, Seoul 140-742, Republic of Korea

E-mail: akkim@sookmyung.ac.kr

Key words: tumor necrosis factor-related apoptosis-inducing ligand, caffeic acid phenethyl ester, combination, hepatoma

\section{Introduction}

Hepatocellular carcinoma (HCC) is the most common primary liver cancer in adults, and is the third leading cause of cancer mortality worldwide (1). Although the highest incidence rates of HCC occur in Asia and Africa, HCC incidence frequency is increasing in developed countries, including the USA and those of Western Europe $(2,3)$. Although there are several treatment options such as surgery, radiation and chemotherapy, most patients suffering from advanced HCC are candidates for palliative treatments only (4). Therefore, novel therapeutic approaches for HCC are required for more effective treatment of this malignancy.

Tumor necrosis factor-related apoptosis-inducing ligand (TRAIL/Apo2L), a member of the tumor necrosis factor (TNF) superfamily, is known to induce apoptosis in various cancer cells, with minimal toxicity to normal cells (5). Due to this selective apoptosis-inducing activity, TRAIL has received great attention as a promising candidate for cancer therapeutics. However, recent studies have demonstrated that many types of malignant cells, including hepatocellular carcinoma cells, are resistant to the apoptotic effect mediated by TRAIL (6). Because TRAIL alone is not sufficient to induce cancer cell death in these resistant cells, recent studies have aimed at overcoming the resistance of cancer cells to TRAIL by combination of sensitizing agents with TRAIL. The combination of chemotherapeutic agents, such as genotoxic drugs, small molecule-inhibitors and natural products, with TRAIL has been shown to be successful in the enhancement of susceptibility to TRAIL-induced cell death in various TRAIL-resistant tumor cells $(7,8)$.

Polyphenolic compounds, a large group of phytochemicals, have been reported to possess anticancer and chemopreventive properties $(9,10)$. Numerous studies have demonstrated that TRAIL, in combination with polyphenols, effectively synergizes TRAIL-induced apoptotic cell death in various malignant cells $(11,12)$.

Here, we examined the potential role of caffeic acid phenethyl ester (CAPE), a polyphenolic compound in honeybee propolis, as a sensitizing agent for restoring the susceptibility of SK-Hepl cells to TRAIL, and demonstrated the underlying mechanisms involved in this enhancement of susceptibility. 


\section{Materials and methods}

Materials. Caffeic acid phenethyl ester (CAPE), MTT (3-(4,5dimethylthiazolyl-2-yl)-2,5-diphenyl-tetrazolium bromide), and 3,3'-dihexyloxacarbocyanine iodide $\left(\mathrm{DiOC}_{6}\right)$ were purchased from Sigma-Aldrich Co. (St. Louis, MO, USA). Soluble recombinant human TRAIL/Apo2 ligand was purchased from PeproTech (Rocky Hill, NJ, USA). Caspase inhibitors were obtained from Santa Cruz Biotechnology (Santa Cruz, CA, USA). Human recombinant DR4/Fc and DR5/Fc chimera protein were purchased from R\&D Systems (Minneapolis, MN, USA). All the antibodies for western blot analysis and MAPK inhibitors were purchased from Cell Signaling (Beverly, MA, USA). Dulbecco's modified Eagle's medium (DMEM), fetal bovine serum (FBS), Dulbecco's phosphate-buffered saline (DPBS), trypsin-EDTA and penicillin/streptomycin were purchased from WelGENE (Daegu, Korea).

Cell culture. Human hepatocellular carcinoma SK-Hep1 cells were obtained from the Korean Cell Line Bank (Seoul, Korea). Cells were cultured at $37^{\circ} \mathrm{C}$ in a humidified condition of $5 \% \mathrm{CO}_{2}$ and maintained in DMEM supplemented with $10 \% \mathrm{FBS}$ and penicillin/streptomycin.

Cell viability assay. MTT assay was used to determine cell viability. Cells were seeded in 96-well plate, incubated for $24 \mathrm{~h}$ and treated as described in individual experiments. After incubation for $24 \mathrm{~h}$, MTT solution was added to each well for $4 \mathrm{~h}$ and the resulting formazan product was dissolved in dimethyl sulfoxide (DMSO). The absorbance was measured at $570 \mathrm{~nm}$ with a microplate reader (EL800, Bio-Tek Instrument Inc., Winooski, VT, USA) and the cell viability (\%) was calculated.

Flow cytometric analysis for mitochondrial membrane potential (MMP). To analyze loss of MMP, treated cells were harvested and incubated with $\mathrm{DiOC}_{6}(40 \mathrm{nM})$ at $37^{\circ} \mathrm{C}$ for $30 \mathrm{~min}$ in the dark. Fluorescence intensity was measured by flow cytometry (FACSCanto II Flow Cytometer, BD Biosciences, USA).

Detection of apoptosis by flow cytometry. Apoptotic cells were quantified by Annexin V/propidium iodide (PI) staining assay. Cells were seeded in 6-well plates for $24 \mathrm{~h}$ and treated as described in individual experiments. After $24 \mathrm{~h}$, cells were harvested and resuspended in binding buffer. Then, cells were incubated with Annexin V-FITC and PI for $15 \mathrm{~min}$ at room temperature in the dark. Apoptotic cell death was evaluated by flow cytometry and the population of Annexin $\mathrm{V}^{+} / \mathrm{PI}^{-}$was considered as apoptotic cells.

Analysis of death receptors on the cell surface. Surface expression of DR4 and DR5 was analyzed by indirect staining with primary mouse anti-DR4 and DR5 followed by phycoerythrin (PE)-conjugated goat anti-mouse IgG1 (Santa Cruz Biotechnology). As a negative control, cells were incubated with a normal mouse IgG1 antibody in the same conditions (Santa Cruz Biotechnology). Death receptor expression was analyzed by flow cytometry.

Western blot analysis. Cells treated as described in individual experiments were lysed with RIPA buffer (50 mM Tris- $\mathrm{HCl}$,
pH 8.0, with $150 \mathrm{mM} \mathrm{NaCl}, 0.1 \% \mathrm{NP}-40,0.5 \%$ sodium deoxycholate and $0.1 \%$ SDS). Equal amount of protein was resolved by $10 \%$ SDS-PAGE and then transferred to polyvinylidene difluoride (PVDF) membranes. The blots were blocked with $5 \%$ non-fat dry milk for $2 \mathrm{~h}$ at room temperature and incubated overnight at $4^{\circ} \mathrm{C}$ with appropriate primary antibodies. Horseradish peroxidase-conjugated anti-rabbit or anti-mouse antibodies were used as secondary antibodies. Protein signals were visualized with enhanced chemiluminescence (ECL) solution and quantified by Multi Gauge software (Fuji Photo Film, Japan).

Statistical analysis. Values are presented as the mean \pm SD. Statistical significance was evaluated by using one-way analysis of variance (ANOVA) followed by Tukey's test. P-value of $<0.05$ was regarded statistically significant.

\section{Results}

Combined CAPE and TRAIL treatments promote TRAILinduced apoptosis in SK-Hepl cells. In order to investigate whether CAPE sensitizes SK-Hep1 cells to TRAIL-induced cell death, we treated cells with TRAIL, in the presence or absence of CAPE for $24 \mathrm{~h}$ at various concentrations. As shown in Fig. 1A, treatment with CAPE or TRAIL alone resulted in a limited inhibition of cell viability $(<20 \%)$ at $24 \mathrm{~h}$. In contrast, cell viability was markedly reduced by combined treatment when cells were treated with a fixed concentration of TRAIL and varying concentrations of CAPE, and vice versa. The CAPE concentration at $30 \mu \mathrm{g} / \mathrm{ml}$ was selected for further study because it effectively induced a significant reduction of cell viability in the combined treatment with TRAIL, with only a slight decrease in the percentage of viable cells. Next, we examined whether the combined effect of CAPE and TRAIL on cell viability is due to increased apoptosis. As shown in Fig. 1B, co-treatment of CAPE and TRAIL significantly enhanced the amount of apoptotic cells (\%), whereas treatment with CAPE or TRAIL alone induced only a slight increase of apoptosis, compared to untreated group. These findings suggest that the combined treatment of CAPE and TRAIL effectively induces apoptotic cell death in SK-Hepl cells.

Combined CAPE and TRAIL treatment potentiates the activation of caspase-mediated death signal. Next, we examined whether caspases are activated by combination of CAPE and TRAIL. As shown in Fig. 2A, CAPE alone did not induce any significant changes of caspases. In cells treated with TRAIL alone, caspase- 3 was partially cleaved to the intermediate inactive fragment $\mathrm{p} 19$, whereas combined treatment with CAPE and TRAIL induced further cleavage into p17, the fully active subunit of caspase-3. Also, cleaved caspase-7 (20 kDa), as the active form of caspase-7, was significantly increased through the combination of CAPE and TRAIL, but not through the administration of either agent alone. We found that PARP, a well known substrate for caspase-3 and caspase-7, was remarkably cleaved by the combined treatment. It is well known that the activation of effector caspases, like caspase-3 and -7 is regulated by initiator caspases, such as caspase- 8 and caspase-9. As shown in Fig. 2A, treatment with a combination of CAPE and TRAIL markedly decreased the levels of 

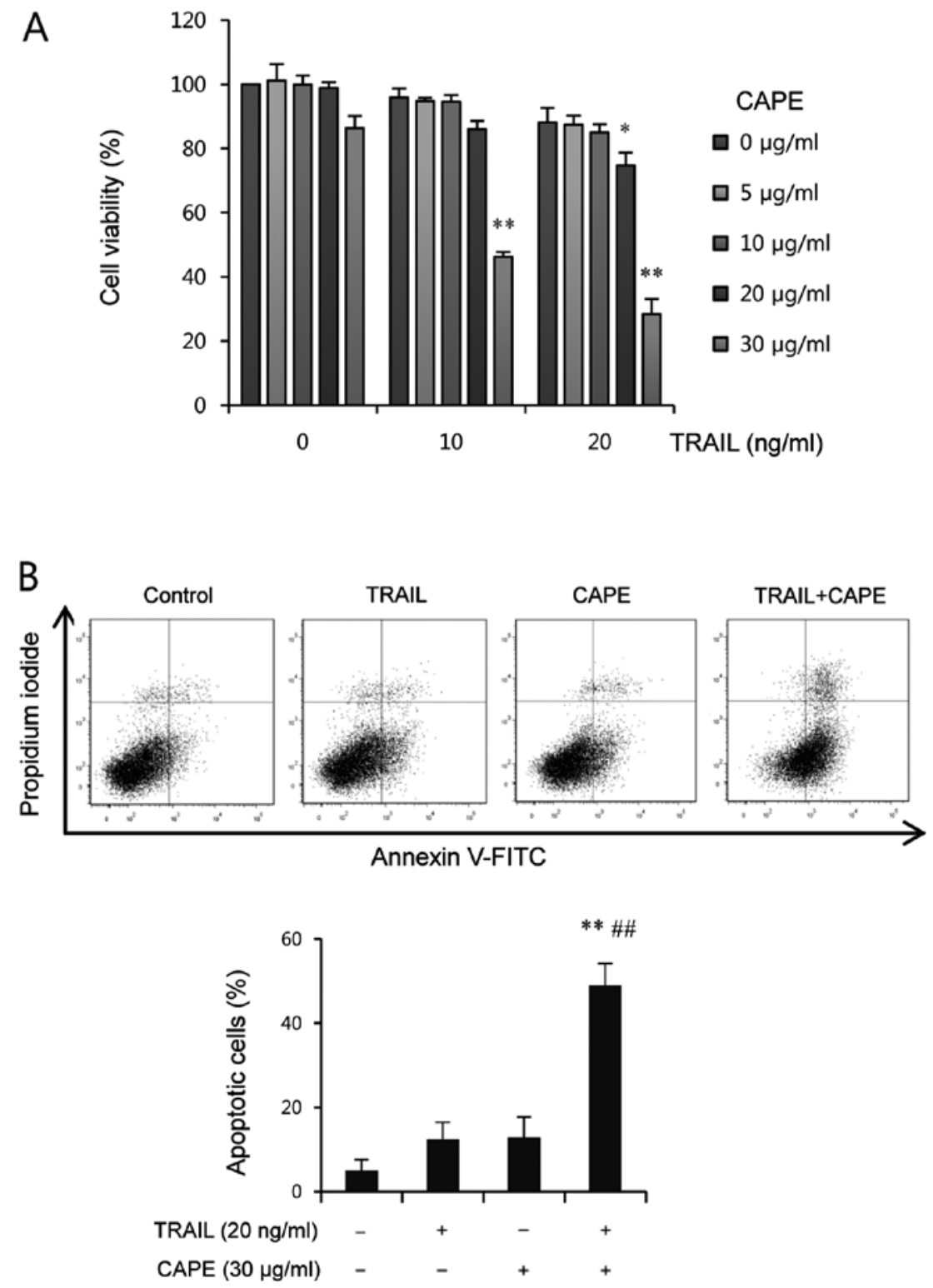

Figure 1. Combination effect of CAPE and TRAIL on TRAIL-induced apoptosis of SK-Hep1 cells. (A) Effect of CAPE and TRAIL on cell viability. Cells were treated with TRAIL alone or in combination with CAPE for $24 \mathrm{~h}$ at the indicated concentrations. Cell viability was evaluated by MTT assay. Data represent the mean \pm SD $(n=3)$. Statistical significance: ${ }^{*} \mathrm{p}<0.01,{ }^{* *} \mathrm{p}<0.001$ vs. control TRAIL-untreated cells. (B) Effect of CAPE on TRAIL-induced apoptosis in SK-Hep1 cells. Cells were treated with CAPE $(30 \mu \mathrm{g} / \mathrm{ml})$ alone, or in combination with TRAIL $(20 \mathrm{ng} / \mathrm{ml})$ for $24 \mathrm{~h}$. Apoptosis was determined by Annexin V/PI dual staining. Representative results are shown as dot plot (upper panel). Data in the bar graph represent the mean $\pm \mathrm{SD}$ of three independent experiments (lower panel). ${ }^{* *} \mathrm{p}<0.001$ vs. untreated control; ${ }^{\# \#} \mathrm{p}<0.001$ vs. cells treated with CAPE or TRAIL alone.

precursor protein of caspase- 8 and caspase-9 compared to either agent alone, indicating the significant activation of these caspases. In certain cells, BH3-interacting domain death agonist (Bid) cleaved into tBid (truncated Bid) by caspase- 8 , which, in turn, induces mitochondrial damage (13). We found that full-length Bid was significantly decreased by combined treatment with CAPE and TRAIL, but not by either agent alone (Fig. 2A).

Next, to investigate the role of caspases in combination-induced apoptosis of SK-Hep1 cells, a broad caspase inhibitor, z-VAD-fmk, was used. As shown in Fig. 2B, pretreatment with z-VAD-fmk completely blocked the enhanced cytotoxicity induced by the combination of CAPE and TRAIL. Annexin V/PI staining assay also showed that
z-VAD-fmk rescued the increase of apoptotic cell death by the combination of CAPE and TRAIL (Fig. 2C). We found that z-VAD-fmk almost completely abolished the combination treatment-induced PARP cleavage, caspase activation, and Bid cleavage (Fig. 2D). Taken together, these results indicate that combination treatment-induced apoptosis is mediated through a caspase-dependent pathway.

Both extrinsic and intrinsic apoptotic pathways are important for apoptosis in response to combined treatment with CAPE and TRAIL. We next used various caspase inhibitors to examine the respective roles of caspases in CAPE and TRAIL combinationinduced apoptosis. As shown in Fig. 2E, the CAPE-triggered enhancement of TRAIL-induced apoptosis was significantly 

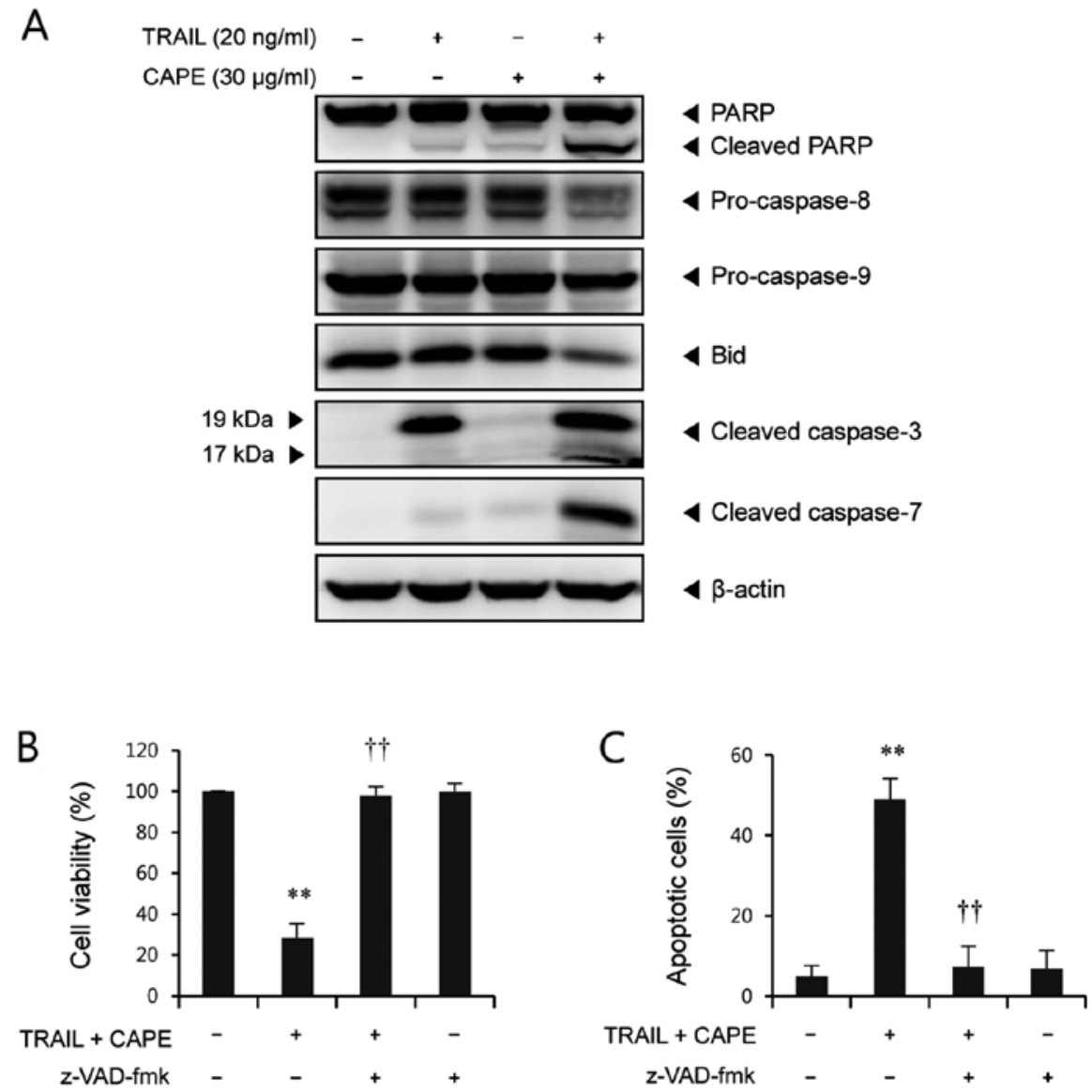

D

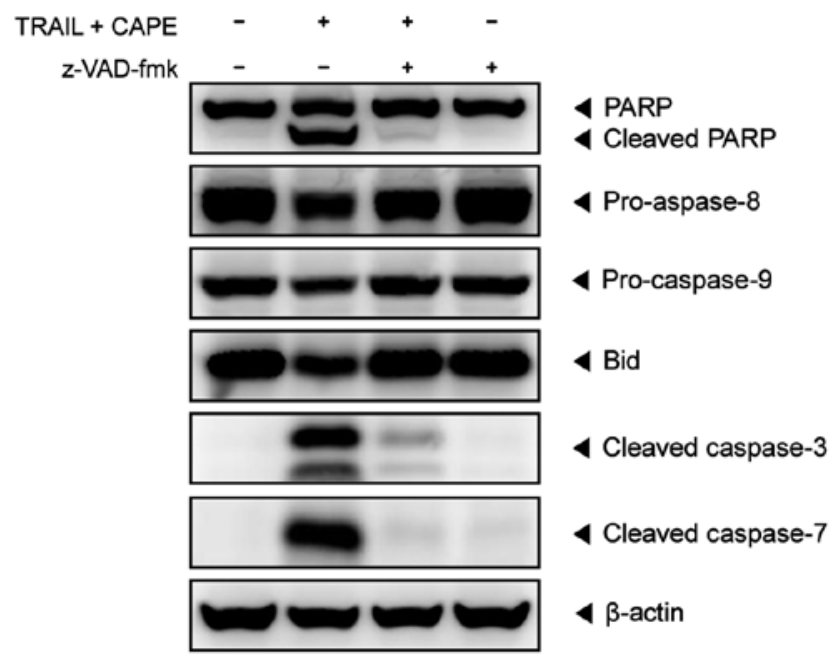

Figure 2. CAPE sensitizes SK-Hep1 cells to TRAIL-induced apoptosis signal via the extrinsic and intrinsic pathways. (A) Effect of combined treatment with CAPE and TRAIL on levels of apoptosis-associated proteins. Cells were treated with TRAIL ( $20 \mathrm{ng} / \mathrm{ml})$ with or without CAPE ( $30 \mu \mathrm{g} / \mathrm{ml})$. After $24 \mathrm{~h}$, PARP cleavage, activation of caspases and Bid cleavage were examined by western blot analysis. (B-D) Cells were pretreated with z-VAD-fmk (20 $\mu \mathrm{M})$ for $30 \mathrm{~min}$, and further treated with combination ( $30 \mu \mathrm{g} / \mathrm{ml}$ CAPE plus $20 \mathrm{ng} / \mathrm{ml}$ TRAIL) for $24 \mathrm{~h}$. The effect of z-VAD-fmk on the combination treatment was evaluated by (B) MTT assay, (C) Annexin V/PI flow cytometry analysis, and (D) western blot analysis. (B and C) ${ }^{* *}$ p $<0.001$ vs. untreated control; ${ }^{\dagger \dagger}$ p $<0.001$ vs. cells treated with CAPE plus TRAIL.

blocked by z-DEVD-fmk (caspase-3/-7 inhibitor), z-IETD-fmk (a caspase-8 inhibitor), and z-LEHD-fmk (a caspase-9 inhibitor), indicating that CAPE enhances TRAIL-induced apoptosis through activation of caspase-3, -7, -8 and -9.
Disruption of mitochondrial membrane potential $\left(\Delta \Psi_{\mathrm{m}}\right)$ is important for the release of apoptotic factors such as cytochrome $c$ and apoptosis-inducing factor (AIF), leading to the induction of the caspase cascade. To further examine 

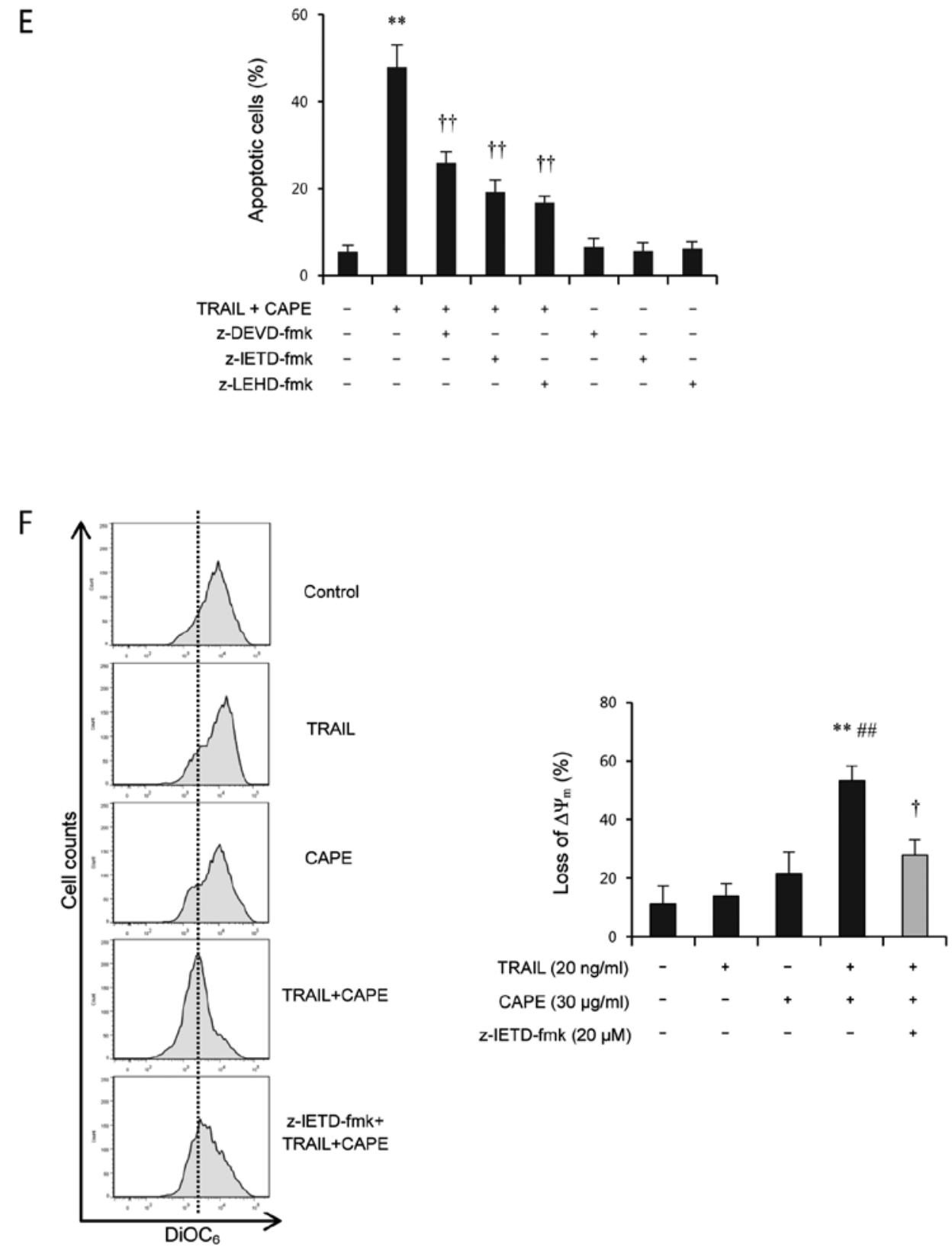

Figure 2. Continued. (E) Cells were pretreated with $20 \mu \mathrm{M}$ caspase-3 inhibitor (z-DEVD-fmk), caspase-8 inhibitor (z-IETD-fmk), or caspase-9 inhibitor (z-LEHD-fmk) for $30 \mathrm{~min}$ and further treated with combination ( $30 \mu \mathrm{g} / \mathrm{ml}$ CAPE plus $20 \mathrm{ng} / \mathrm{ml}$ TRAIL) for $24 \mathrm{~h}$. Apoptotic cell death (\%) was evaluated by Annexin V/PI flow cytometry analysis. Statistical significance: ${ }^{* *} \mathrm{p}<0.001$ vs. untreated control; ${ }^{\dagger \dagger} \mathrm{p}<0.001$ vs. cells treated with CAPE plus TRAIL. (F) Effect of combined treatment with CAPE and TRAIL on mitochondrial membrane potential. Cells were pretreated with z-IETD-fmk $(20 \mu \mathrm{M})$ for $30 \mathrm{~min}$, and further treated with combination ( $30 \mu \mathrm{g} / \mathrm{ml} \mathrm{CAPE}$ plus $20 \mathrm{ng} / \mathrm{ml}$ TRAIL) for $24 \mathrm{~h}$. Cells were stained with $\operatorname{DiOC}_{6}(40 \mathrm{nM})$ and the accumulation of DiOC was analyzed $^{2}$ by flow cytometry. A representative result is shown (left). The loss of $\Delta \Psi_{\mathrm{m}}(\%)$ is presented as a bar graph (right). The bar represents the mean $\pm \mathrm{SD}(\mathrm{n}=3$ ). Statistical significance: ${ }^{* * *} \mathrm{p}<0.001$ vs. untreated control; ${ }^{\# \#} \mathrm{p}<0.001$ vs. cells treated with CAPE or TRAIL alone; ${ }^{\dagger} \mathrm{p}<0.01$ vs. cells treated with CAPE plus TRAIL.

the role of the intrinsic death pathway, we measured $\Delta \Psi_{\mathrm{m}}$ by staining cells with $\mathrm{DiOC}_{6}$ fluorescence, which labels mitochondria. As shown in Fig. 2F, CAPE or TRAIL alone slightly induced loss of $\Delta \Psi_{\mathrm{m}}$. In contrast, combination of CAPE with TRAIL led to the significant depolarization of the mitochondrial membrane, confirming the involvement of the mitochondria-dependent intrinsic apoptosis pathway. Moreover, z-IETD-fmk, a caspase- 8 specific inhibitor, significantly rescued the alteration of $\Delta \Psi_{\mathrm{m}}$ by combined treatment, suggesting the important role of caspase- 8 as an upstream regulator of mitochondria-mediated apoptosis.
These results indicate that both intrinsic and extrinsic apoptotic pathways are involved in combined treatment-induced apoptosis.

CAPE potentiates TRAIL-induced apoptosis through DR4 and DR5 upregulation. It is well known that TRAIL interacts with its receptors, DR4 and DR5, to trigger apoptotic cell death (14). To investigate whether enhanced apoptosis by combination treatment is associated with this signaling pathway, we examined the effect of CAPE on the surface expression of DR4 and DR5. We found that CAPE treat- 
A

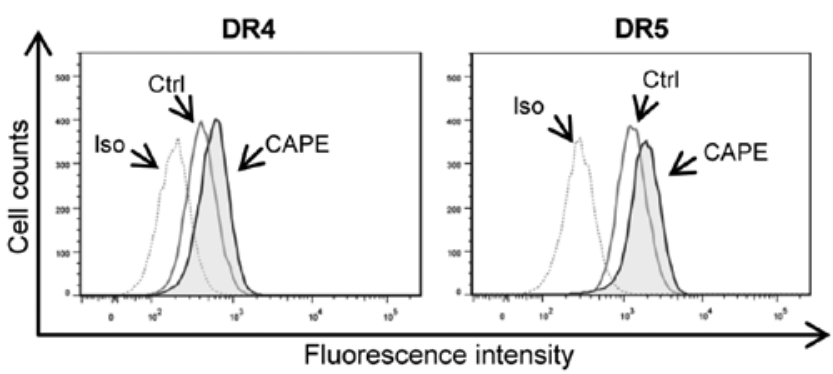

B

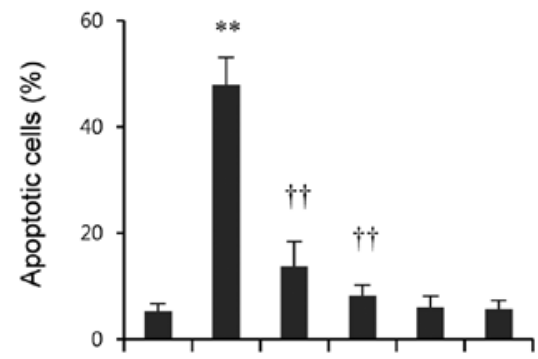

TRAIL + CAPE DR4/Fc chimera DR5/Fc chimera

C

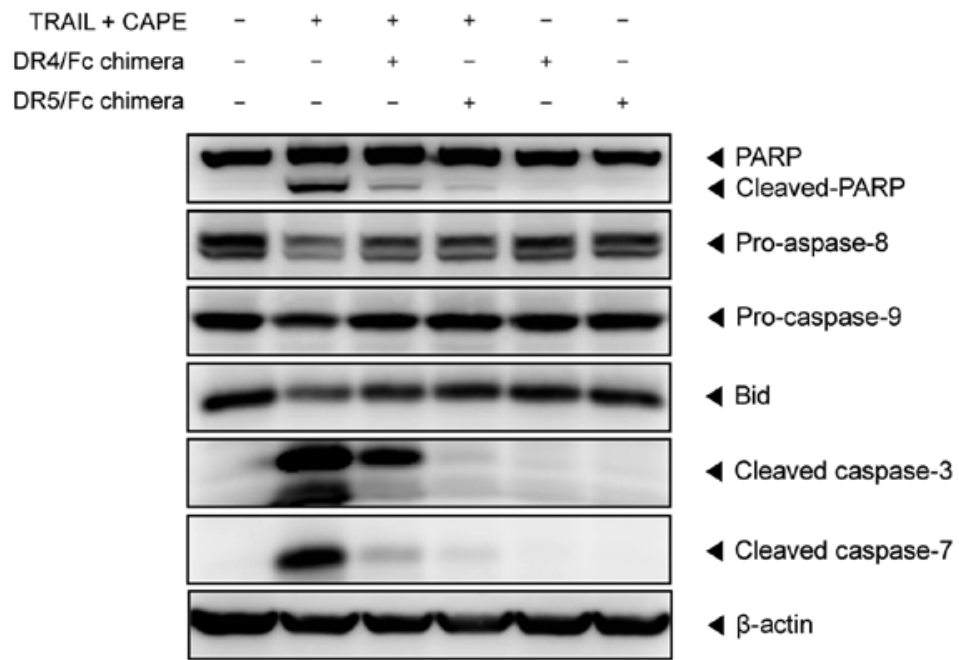

Figure 3. The role of death receptors in apoptosis induced by CAPE and TRAIL combination. (A) Cells were treated with CAPE (30 $\mu \mathrm{g} / \mathrm{ml}$ ) for $24 \mathrm{~h}$ and the cell surface expression of DR4 and DR5 was analyzed by flow cytometry after staining with anti-DR4 or anti-DR5 antibodies. Isotype-matched non-binding antibodies (Iso) were used as a negative control. (B and C) Cells were treated with $30 \mu \mathrm{g} / \mathrm{ml} \mathrm{CAPE}$ and $20 \mathrm{ng} / \mathrm{ml}$ TRAIL for $24 \mathrm{~h}$ in the absence or presence of DR4/Fc $(100 \mathrm{ng} / \mathrm{ml})$ or DR5/Fc chimera protein $(100 \mathrm{ng} / \mathrm{ml})$ prior to $(\mathrm{B})$ Annexin V/PI double staining assay and $(\mathrm{C})$ western blot analysis. $(\mathrm{B}){ }^{* *}$ p $<0.001 \mathrm{vs}$. untreated control; ${ }^{\dagger} \mathrm{p}<0.001$ vs. cells treated with CAPE plus TRAIL.

ment upregulated both DR4 and DR5 expression (Fig. 3A). Next, to determine the role of these death receptors in the enhancement of TRAIL-induced apoptosis by CAPE, we used DR4/Fc and DR5/Fc chimera antibodies. As shown in Fig. 3B, both DR4 and DR5 blocking by specific chimera protein significantly suppressed the combination treatment-induced apoptosis. Furthermore, combination treatment-induced PARP cleavage, activation of caspases, and Bid cleavage were markedly attenuated by DR4/Fc and DR5/Fc chimera proteins (Fig. 3C). These results indicate that the upregulation of DR4 and DR5 is essential for the sensitizing effect of CAPE on TRAIL-mediated apoptosis.

Treatment with CAPE alone or together with TRAIL effectively activates p38 MAPK but suppresses JNK phosphorylation. We investigated whether the mitogen-activated protein kinase (MAPK) signaling pathway is affected by the combination effect of CAPE and TRAIL on SK-Hep1 cells. As shown in Fig. 4A, p38 was significantly activated after $16 \mathrm{~h}$ of CAPE treatment, whereas JNK activation was strongly suppressed 
A

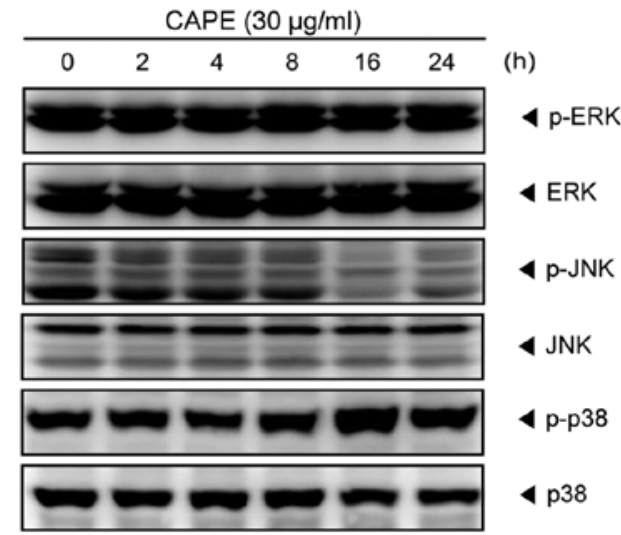

B TRAIL $(20 \mathrm{ng} / \mathrm{ml})$
CAPE $(30 \mu \mathrm{g} / \mathrm{ml})$

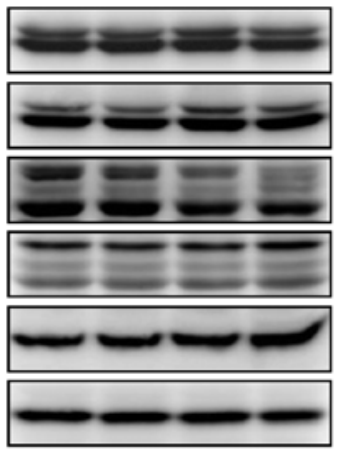

4 p-ERK

4 ERK

4 p-JNK

4 JNK

4 p-p38

4 p38
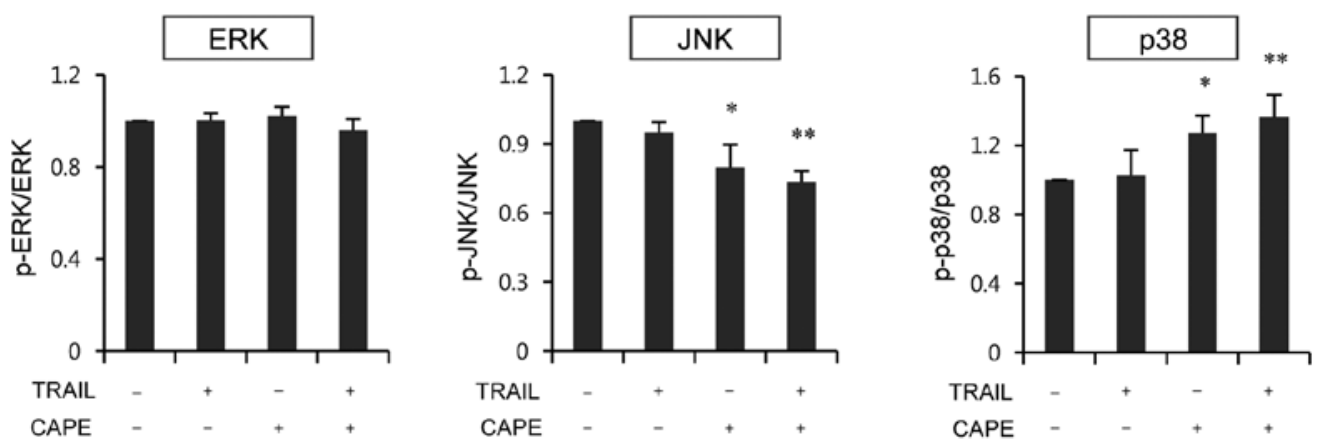

Figure 4. Effect of CAPE in the presence or absence of TRAIL on MAPK family members. (A) Cells were treated with $30 \mu \mathrm{g} / \mathrm{ml}$ CAPE for various time points and cell lysates were analyzed by western blot analysis for phosphorylated ERK, JNK and p38. Antibodies against total ERK, total JNK and total p38 were used to normalize protein loading. (B) Cells were treated with CAPE $(30 \mu \mathrm{g} / \mathrm{ml})$ with or without TRAIL $(20 \mathrm{ng} / \mathrm{ml})$. After $24 \mathrm{~h}$, cell lysates were analyzed by western blot analysis for phosphorylated ERK, JNK and p38. Antibodies against total ERK, total JNK and total p38 were used to normalize protein loading. Representative data are shown in the upper panel. Protein band intensity was measured by image analyzer and presented as bar graphs (lower panel). The bar represents the mean \pm SD $(\mathrm{n}=3)$. Statistical significance: ${ }^{*} \mathrm{p}<0.05,{ }^{* *} \mathrm{p}<0.001$ vs. untreated control.

after $16 \mathrm{~h}$ of treatment. However, CAPE had no significant effect on ERK activation. Combination of CAPE and TRAIL showed similar trends in relation to their effect on MAPK phosphorylation (Fig. 4B). Interestingly, the activation of p38 and the inhibition of JNK were further enhanced by combination treatment, as compared to treatment with CAPE alone. The level of ERK was not changed by either single agents or by combined treatment of CAPE and TRAIL.

p38 MAPK pathway is involved in the upregulation of DR4 and DR5 and subsequent apoptosis. To investigate the possible role of p38 on combination treatment-induced apoptosis, cells were pretreated with SB203580, a specific inhibitor of p38, and apoptosis was evaluated by Annexin V/PI double staining assay. As shown in Fig. 5A, pretreatment with SB203580 significantly blocked the combination-induced apoptosis, suggesting the involvement of p38 in combination-mediated apoptosis. Furthermore, we also examined whether inhibition of p38 attenuates the upregulation of DR4 and DR5 by CAPE treatment. As shown in Fig. 5B, CAPE resulted in a significant upregulation of DR4 and DR5, and pretreatment of SB203580 suppressed both DR4 and DR5 expression. These results indicate the important role of the p38 MAPK pathway on DR4 and DR5 upregulation, and on the subsequent induction of apoptosis. 

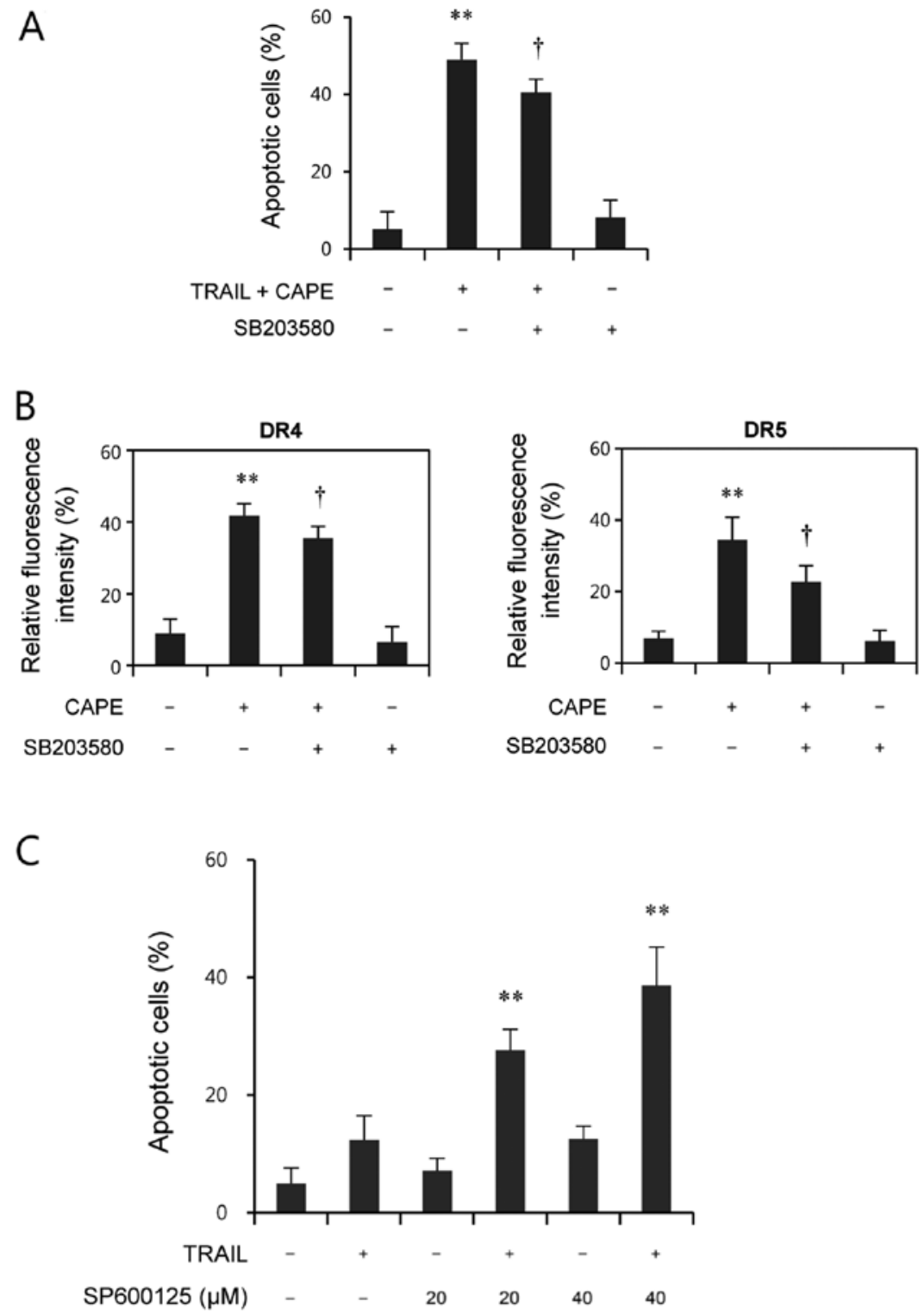

D
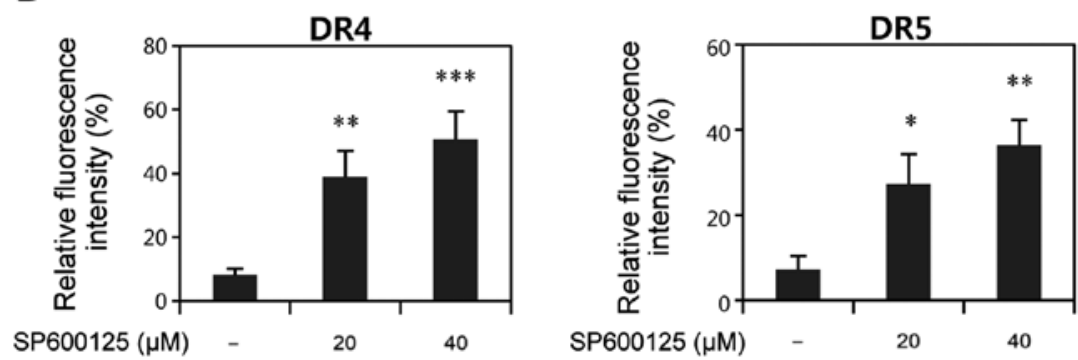

Figure 5. Effect of SB203580 and SP600125 on the regulation of death receptors and apoptosis induced by CAPE and TRAIL. (A) Cells were pretreated with $20 \mu \mathrm{M}$ SB203580 for $30 \mathrm{~min}$ and then treated with $30 \mu \mathrm{g} / \mathrm{ml}$ CAPE and $20 \mathrm{ng} / \mathrm{ml}$ TRAIL for $24 \mathrm{~h}$. Apoptotic cell death (\%) was evaluated by flow cytometry. The bar represents the mean $\pm \mathrm{SD}(\mathrm{n}=3)$. Statistical significance: ${ }^{* *} \mathrm{p}<0.001$ vs. untreated control; ${ }^{\dagger} \mathrm{p}<0.05$ vs. cells treated with CAPE plus TRAIL. (B) Cells were pretreated with $20 \mu \mathrm{M} \mathrm{SB} 203580$ for $30 \mathrm{~min}$ and then treated with $30 \mu \mathrm{g} / \mathrm{ml}$ CAPE for $24 \mathrm{~h}$ and the expression of DR4 and DR5 was analyzed by flow cytometry after staining with anti-DR4 or anti-DR5 antibodies. The bar represents the mean $\pm \mathrm{SD}(\mathrm{n}=3)$. Statistical significance: ${ }^{* * *} \mathrm{p}<0.001$ vs. untreated control; ${ }^{\dagger} \mathrm{p}<0.05 \mathrm{vs}$. cells treated with CAPE. (C) Cells were pretreated with SP600125 (20-40 $\mu \mathrm{M})$ for $30 \mathrm{~min}$ before TRAIL $(20 \mathrm{ng} / \mathrm{ml})$ for $24 \mathrm{~h}$. Apoptotic cell death (\%) was analyzed by flow cytometry. Statistical significance: ${ }^{* * *}$ p $<0.001$ vs. untreated control. (D) Cells were pretreated with SP600125 (20-40 $\left.\mu \mathrm{M}\right)$ for $30 \mathrm{~min}$ and incubated for $24 \mathrm{~h}$. The expression of DR4 and DR5 was analyzed by flow cytometry. Statistical significance: ${ }^{*} \mathrm{p}<0.05,{ }^{* *} \mathrm{p}<0.01,{ }^{* * *} \mathrm{p}<0.001$ vs. untreated control

JNK inhibition upregulates DR4 and DR5 and enhances TRAIL-induced apoptosis. JNK activation has been previously reported to contribute to TRAIL resistance in HCC cells (6).
To assess the effect of JNK inhibition on TRAIL-induced apoptosis, a JNK specific inhibitor, SP600125, was used. As shown in Fig. 5C, combination of TRAIL with SP600125, 
as in the case of the combination of TRAIL with CAPE, significantly enhanced TRAIL-induced apoptosis. We also evaluated the effect of JNK inhibition on DR4 and DR5 surface expression. As shown in Fig. 5D, SP600125 effectively upregulated DR4 and DR5 surface expression. Taken together, these results suggest that the CAPE-induced inhibition of JNK may contribute to the sensitizing effect of CAPE on TRAIL-resistant SK-Hep1 cells.

\section{Discussion}

CAPE is an active phenolic component of honeybee propolis, and has been reported to possess a broad spectrum of biological activities, including anticancer and apoptosis inducing properties $(15,16)$. Propolis, a resin-like mixture produced by honeybees, is a concentrated source of polyphenolic compounds (17). Previous reports have shown the synergistic induction of TRAIL-induced cancer cell death by combination of TRAIL with propolis extracts or polyphenolic compounds isolated from propolis $(18,19)$. The molecular mechanisms to overcome TRAIL-resistance by combination with these polyphenols in propolis have reported for artepillin C, chrysin, apigenin, and naringenin (20-23). However, the underlying mechanisms by which CAPE sensitizes TRAIL-resistant malignant cells have not yet been fully elucidated. In the present study, we demonstrated that treatment with CAPE effectively sensitized SK-Hep1 cells to TRAIL-mediated apoptosis.

Caspases belong to a family of cysteine proteases which play a pivotal role in apoptotic cell death. Activation of the caspase cascade is mediated by two major pathways: one is the death receptor-mediated (extrinsic) pathway, and the other is the mitochondria-mediated (intrinsic) pathway (24). Apoptosis by death receptors is initiated by binding of the death-inducing ligands to its receptors, leading to the formation of death-inducing signaling complexes (DISC) and the subsequent activation of caspase- 8 . Active caspase- 8 initiates the caspase cascade by direct activation of effector caspases, such as caspase-3 and -7 (25). In the mitochondria-dependent pathway, intrinsic apoptotic stimuli trigger mitochondria membrane permeabilization and the subsequent release of cytochrome $c$, which activates caspase- 9 by forming the apoptosome (26). In some cells, Bid is cleaved to tBid by caspase- 8 and serves as a molecular link between the extrinsic and intrinsic pathways by inducing release of mitochondrial proteins (13). In our results, the combination of CAPE and TRAIL enhanced the activation of effector caspases (caspase-3, -7) and their substrate PARP cleavage. We also observed the activation of caspase- 9 as well as the activation of capsase- 8 and Bid cleavage in combination-treated cells. Furthermore, such activation of apoptosis-related proteins by combination treatment was strongly inhibited by the pan-caspase inhibitor, z-VAD-fmk, suggesting the critical role of caspases in combination-treated cells. Moreover, pretreatment with z-IETD-fmk (caspase- 8 inhibitor) and z-LEHD-fmk (caspase-9 inhibitor) resulted in a significant inhibition of combination-induced apoptosis, indicating the involvement of extrinsic and intrinsic apoptotic pathways. Involvement of mitochondria-mediated apoptosis was also confirmed by the marked increase of mitochondrial membrane depolarization in response to combination treatment with CAPE and TRAIL.

TRAIL-induced death signal is initiated by the binding of TRAIL with its two death receptors DR4 (TRAIL-R1) and DR5 (TRAIL-R2), leading to induction of the extrinsic apoptosis pathway (27). Therefore, targeting of DR4 and DR5 in TRAIL-resistant cells seems to be a useful strategy to sensitize cancer cells to TRAIL-induced apoptosis. Previous reports have shown that a combination of agents which upregulate DR4 and/or DR5 TRAIL receptors induced a synergistic apoptotic activation in TRAIL-resistant tumor cells $(28,29)$. In this study, we found that upregulation of DR4 and DR5 by CAPE was critical for the CAPE-mediated enhancement of TRAIL-induced apoptosis. Blocking of TRAIL binding with DR4 and DR5 death receptors by their respective dominant-negative chimeric proteins markedly suppressed the effect of CAPE on TRAIL-induced apoptosis. However, DR5/Fc chimera was more effective in reducing apoptosis than DR4/Fc blocking protein. In agreement with our results, several reports have shown the prominent role of DR5 over that of DR4 in TRAIL-mediated apoptosis $(30,31)$.

We also investigated whether MAPKs are involved in the CAPE-induced sensitization of SK-Hep1 cells to TRAIL-induced apoptosis. The MAPK pathways participate in the regulation of a wide variety of cellular processes, such as growth, proliferation, differentiation, stress response, survival, and apoptosis $(32,33)$. In this study, we investigated the roles of p38 and JNK in the sensitizing effect of CAPE on TRAIL-induced apoptosis in SK-Hep1 cells, and found that p38 and JNK played opposing roles. Our results showed that the p38 inhibitor, SB203580, significantly attenuated CAPE-induced DR4 and DR5 expression, and abolished the combination treatment-induced apoptosis. These results regarding the upregulation of TRAIL receptors through p38 activation are consistent with those of previous studies regarding diosgenin (34), damnacanthal (35) and lupulone (36).

Mucha et al have reported that the frequent overexpression of JNK in HCC cells contributes to TRAIL-resistance in these cells (6). They showed that JNK inhibition by SP600125 greatly sensitized hepatoma cells to TRAIL-induced apoptosis, without affecting normal cells, suggesting the novel strategy of targeting JNK for effective TRAIL-based cancer therapy. In our results, we found that JNK phosphorylation was significantly reduced by CAPE alone, or in combination with TRAIL. Consistent with the results of Mucha et al, combination of SP600125 and TRAIL enhanced TRAILmediated apoptosis in SK-Hep1 cells. Moreover, SP600125 significantly upregulated the expression of DR4 and DR5, just like CAPE, indicating that inhibition of JNK by CAPE resulted in the upregulation of DRs, and the subsequent enhancement of TRAIL-induced apoptosis in SK-Hep1 cells.

In summary, our study showed that CAPE effectively augmented TRAIL-induced apoptosis in SK-Hep1 cells through caspase-dependent extrinsic and intrinsic apoptotic pathways. The upregulation of death receptors by CAPE treatment was involved in p38 activation and JNK inhibition. Collectively, our results demonstrated a novel function of CAPE in TRAIL-induced apoptosis in HCC cells. 


\section{Acknowledgements}

This work was supported by Basic Science Research Program through the National Research Foundation (NRF) funded by the Korea government (MEST) (2010-0010538) and the NRF grant funded by the Korea government (2011-0030699).

\section{References}

1. Schutte K, Bornschein J and Malfertheiner P: Hepatocellular carcinoma-epidemiological trends and risk factors. Dig Dis 27: 80-92, 2009.

2. McGlynn KA, Tsao L, Hsing AW, Devesa SS and Fraumeni JF Jr: International trends and patterns of primary liver cancer. Int $\mathrm{J}$ Cancer 94: 290-296, 2001.

3. Harnois DM: Hepatitis $\mathrm{C}$ virus infection and the rising incidence of hepatocellular carcinoma. Mayo Clin Proc 87: 7-8, 2012.

4. Paul SB, Manjunatha YC and Acharya SK: Palliative treatment in advanced hepatocellular carcinoma: has it made any difference? Trop Gastroenterol 30: 125-134, 2009.

5. Ashkenazi A, Pai RC, Fong S, et al: Safety and antitumor activity of recombinant soluble Apo2 ligand. J Clin Invest 104: 155-162, 1999.

6. Mucha SR, Rizzani A, Gerbes AL, et al: JNK inhibition sensitises hepatocellular carcinoma cells but not normal hepatocytes to the TNF-related apoptosis-inducing ligand. Gut 58: 688-698, 2009.

7. Voelkel-Johnson C: TRAIL-mediated signaling in prostate, bladder and renal cancer. Nat Rev Urol 8: 417-427, 2011.

8. Hellwig CT and Rehm M: TRAIL signaling and synergy mechanisms used in TRAIL-based combination therapies. Mol Cancer Ther 11: 3-13, 2012.

9. Spagnuolo C, Russo M, Bilotto S, Tedesco I, Laratta B and Russo GL: Dietary polyphenols in cancer prevention: the example of the flavonoid quercetin in leukemia. Ann NY Acad Sci 1259: 95-103, 2012.

10. Stagos D, Amoutzias GD, Matakos A, Spyrou A, Tsatsakis AM and Kouretas D: Chemoprevention of liver cancer by plant polyphenols. Food Chem Toxicol 50: 2155-2170, 2012.

11. Siddiqui IA, Malik A, Adhami VM, et al: Green tea polyphenol EGCG sensitizes human prostate carcinoma LNCaP cells to TRAIL-mediated apoptosis and synergistically inhibits biomarkers associated with angiogenesis and metastasis. Oncogene 27: 2055-2063, 2008.

12. Jacquemin G, Shirley S and Micheau O: Combining naturally occurring polyphenols with TNF-related apoptosis-inducing ligand: a promising approach to kill resistant cancer cells? Cell Mol Life Sci 67: 3115-3130, 2010.

13. Luo X, Budihardjo I, Zou H, Slaughter C and Wang X: Bid, a $\mathrm{Bcl} 2$ interacting protein, mediates cytochrome c release from mitochondria in response to activation of cell surface death receptors. Cell 94: 481-490, 1998.

14. Wang S and El-Deiry WS: TRAIL and apoptosis induction by TNF-family death receptors. Oncogene 22: 8628-8633, 2003.

15. Grunberger D, Banerjee R, Eisinger K, et al: Preferential cytotoxicity on tumor cells by caffeic acid phenethyl ester isolated from propolis. Cell Mol Life Sci 44: 230-232, 1988.

16. Ozturk G, Ginis Z, Akyol S, Erden G, Gurel A and Akyol O: The anticancer mechanism of caffeic acid phenethyl ester (CAPE): review of melanomas, lung and prostate cancers. Eur Rev Med Pharmacol Sci 16: 2064-2068, 2012.

17. Khalil ML: Biological activity of bee propolis in health and disease. Asian Pac J Cancer Prev 7: 22-31, 2006.

18. Szliszka E, Czuba ZP, Domino M, Mazur B, Zydowicz G and Krol W: Ethanolic extract of propolis (EEP) enhances the apoptosis-inducing potential of TRAIL in cancer cells. Molecules 14: 738-754, 2009
19. Szliszka E, Czuba ZP, Bronikowska J, Mertas A, Paradysz A and Krol W: Ethanolic extract of propolis augments TRAIL-induced apoptotic death in prostate cancer cells. Evid Based Complement Alternat Med 2011: 535172, 2011.

20. Szliszka E, Zydowicz G, Mizgala E and Krol W: Artepillin C (3,5-diprenyl-4-hydroxycinnamic acid) sensitizes LNCaP prostate cancer cells to TRAIL-induced apoptosis. Int J Oncol 41: 818-828, 2012.

21. Li X, Wang JN, Huang JM, et al: Chrysin promotes tumor necrosis factor (TNF)-related apoptosis-inducing ligand (TRAIL) induced apoptosis in human cancer cell lines. Toxicol In Vitro 25: 630-635, 2011.

22. Kim EY, Yu JS, Yang M and Kim AK: Sub-toxic dose of apigenin sensitizes HepG2 cells to TRAIL through ERK-dependent up-regulation of TRAIL receptor DR5. Mol Cells 35: 32-40, 2013.

23. Jin CY, Park C, Hwang HJ, et al: Naringenin up-regulates the expression of death receptor 5 and enhances TRAIL-induced apoptosis in human lung cancer A549 cells. Mol Nutr Food Res 55: 300-309, 2011.

24. Nicholson DW: Caspase structure, proteolytic substrates, and function during apoptotic cell death. Cell Death Differ 6: 1028-1042, 1999.

25. Thorburn A: Death receptor-induced cell killing. Cell Signal 16: 139-144, 2004.

26. Kim HE, Du F, Fang M and Wang X: Formation of apoptosome is initiated by cytochrome c-induced dATP hydrolysis and subsequent nucleotide exchange on Apaf-1. Proc Natl Acad Sci USA 102: 17545-17550, 2005.

27. Zhang L and Fang B: Mechanisms of resistance to TRAIL-induced apoptosis in cancer. Cancer Gene Ther 12: 228-237, 2004.

28. Kauntz H, Bousserouel S, Gosse F and Raul F: The flavonolignan silibinin potentiates TRAIL-induced apoptosis in human colon adenocarcinoma and in derived TRAIL-resistant metastatic cells. Apoptosis 17: 797-809, 2012.

29. Prasad S, Yadav VR, Kannappan R and Aggarwal BB: Ursolic acid, a pentacyclin triterpene, potentiates TRAIL-induced apoptosis through p53-independent up-regulation of death receptors: evidence for the role of reactive oxygen species and JNK. J Biol Chem 286: 5546-5557, 2011.

30. Kelley RF, Totpal K, Lindstrom SH, et al: Receptor-selective mutants of apoptosis-inducing ligand $2 /$ tumor necrosis factor-related apoptosis-inducing ligand reveal a greater contribution of death receptor (DR) 5 than DR4 to apoptosis signaling. J Biol Chem 280: 2205-2212, 2005.

31. Gupta SC, Reuter S, Phromnoi K, et al: Nimbolide sensitizes human colon cancer cells to TRAIL through reactive oxygen species- and ERK-dependent up-regulation of death receptors, p53, and Bax. J Biol Chem 286: 1134-1146, 2011.

32. Raman M, Chen W and Cobb MH: Differential regulation and properties of MAPKs. Oncogene 26: 3100-3112, 2007.

33. Pimienta G and Pascual J: Canonical and alternative MAPK signaling. Cell Cycle 6: 2628-2632, 2007.

34. Lepage C, Leger DY, Bertrand J, Martin F, Beneytout JL and Liagre B: Diosgenin induces death receptor-5 through activation of $\mathrm{p} 38$ pathway and promotes TRAIL-induced apoptosis in colon cancer cells. Cancer Lett 301: 193-202, 2011.

35. Lin FL, Hsu JL, Chou CH, Wu WJ, Chang CI and Liu HJ: Activation of p38 MAPK by damnacanthal mediates apoptosis in SKHep 1 cells through the DR5/TRAIL and TNFR1/ TNF-alpha and p53 pathways. Eur J Pharmacol 650: 120-129, 2011.

36. Lamy V, Bousserouel S, Gosse F, Minker C, Lobstein A and Raul F: Lupulone triggers p38 MAPK-controlled activation of p53 and of the TRAIL receptor apoptotic pathway in human colon cancer-derived metastatic cells. Oncol Rep 26: 109-114, 2011. 